\title{
BMJ Open End-of-life management protocol offered within emergency room (EMPOWER): study protocol for a multicentre study
}

\author{
Rakhee Yash Pal, ${ }^{1}$ Win Sen Kuan (D) , ${ }^{1,2}$ Ling Tiah, ${ }^{3}$ Ranjeev Kumar, ${ }^{4}$ \\ Yoko Kin Yoke Wong, ${ }^{5}$ Luming Shi, ${ }^{5}$ Charles Qishi Zheng, ${ }^{5}$ Jingping Lin, ${ }^{1}$ \\ Sufang Liang, ${ }^{1}$ Uma Chandra Segara, ${ }^{1}$ Woon Chai Yong, ${ }^{6,7}$ \\ Noreen Guek Cheng Chan, 6,7 Mui Teng Chua (D) ,1,2 Irwani Ibrahim ${ }^{1,2}$
}

To cite: Yash Pal R, Kuan WS, Tiah L, et al. End-of-life management protocol offered within emergency room (EMPOWER): study protocol for a multicentre study. BMJ Open 2020;10:e036598. doi:10.1136/ bmjopen-2019-036598

- Prepublication history and additional material for this paper are available online. To view these files, please visit the journal online ().

Received 20 December 2019 Revised 20 March 2020 Accepted 09 April 2020
Check for updates

(C) Author(s) (or their employer(s)) 2020. Re-use permitted under CC BY-NC. No commercial re-use. See rights and permissions. Published by BMJ.

For numbered affiliations see end of article.

Correspondence to Dr Mui Teng Chua; mui_teng_chua@nuhs.edu.sg

\section{ABSTRACT}

Background Patients at their end-of-life (EOL) phase frequently visit the emergency department (ED) due to their symptoms, yet the environment and physicians in ED are not traditionally equipped or trained to provide palliative care. This multicentre study aims to measure the current quality of EOL care in ED to identify gaps, formulate improvements and implement the improved EOL care protocol. We shall also evaluate healthcare resource utilisation and its associated costs.

Methods and analysis This study employs a quasiexperimental interrupted time series design using both qualitative and quantitative methods, involving the EDs of three tertiary hospitals in Singapore, over a period of 3 years. There are five phases in this study: (1) retrospective chart reviews of patients who died within 5 days of ED attendance; (2) pilot phase to validate the CODE questionnaire in the local context; (3) preimplementation phase; (4) focus group discussions (FGDs); and (5) postimplementation phase. In the prospective cohort, patients who are actively dying or have high likelihood of mortality this admission, and whose goal of care is palliation, will be eligible for inclusion. At least 140 patients will be recruited for each preimplementation and postimplementation phase. There will be face-to-face interviews with patients' family members, review of medical records and selfadministered staff survey to evaluate existing knowledge and confidence. The FGDs will involve hospital and community healthcare providers. Data obtained from the retrospective cohort, preimplementation phase and FGDs will be used to guide prospective improvement and protocol changes. Patient, family and staff relevant outcomes from these changes will be measured using time series regression.

Ethics and dissemination The study protocol has been reviewed and ethics approval obtained from the National Healthcare Group Domain Specific Review Board, Singapore. The results from this study will be actively disseminated through manuscript publications and conference presentations.

Trial registration number NCT03906747.

\section{Strengths and limitations of this study}

- This novel multicentre study evaluating end-of-life (EOL) care provided in the emergency department (ED) will be able to evaluate the quality of clinical management rendered to EOL patients via a multiprong approach, from clinical charts and perceptions of family members who were at the bedside.

- By involving our community partners, we shall also be able to identify difficulties faced in caring for these patients in a non-institutional setting.

- Results of this study have the potential to identify current gaps and barriers and inform emergency physicians globally on best practices in providing EOL care in the ED.

- Some limitations to the study design include response rates to questionnaires, selection bias due to non-probability sampling and information bias from retrospective chart reviews

\section{BACKGROUND}

In a report by the Institute of Medicine in 1997, a decent or good death is one that should be free of unnecessary distress and suffering for both patients and their caregivers and should be in general accord with their wishes, within reasonable consistency with clinical, cultural and ethical standards. ${ }^{1}$ The WHO defines palliative care as a multidimensional approach to improve the quality of life for dying patients and their families by caring for the physical, psychosocial and spiritual needs. Emergency physicians, being at the forefront of medicine and healthcare delivery, frequently encounter death as a daily occurrence. Globally, it is estimated that at least 35 million $(60 \%)$ out of 58 million people who are dying annually suffer from advanced illnesses and would benefit from palliative care services for better quality of 
life. $^{2}$ In addition, up to $80 \%$ of terminally ill patients prefer to die at home and yet only a small percentage are able to achieve this. ${ }^{34}$

As the first point of contact in the healthcare system, the emergency department (ED) is particularly important for provision of appropriate end-of-life (EOL) care, especially when a majority of EOL patients use emergency services in the last month of their lives. ${ }^{5}$ Many patients present to the ED due to symptoms of distress, poorly controlled symptoms at home or families who are unable to provide care as a result of physical and mental distress. ${ }^{6}$ Traditionally, the ED has not been regarded as an optimal place for EOL care, due to its inherent nature of overcrowding and chaos, resulting in lack of tranquil infrastructure for families to spend meaningful time with their loved ones during their last moments. ${ }^{47}$ ED physicians are also perceived to lack knowledge and confidence in managing such patients as palliative care contradicts their conventional training on aggressive resuscitation measures. The initiation of palliative care from ED by emergency physicians was found to be at a dismal rate of $18 \%$ in one study. ${ }^{8}$ This highlights the need for more specialised training for emergency physicians to adequately manage the increasing number of patients facing progressive illnesses and disabilities towards the end of their lives.

Palliative care services in Singapore have grown and developed over the past 30 years and is acknowledged as an essential specialty in the healthcare system. ${ }^{9}$ However, palliative care development in ED is limited, ${ }^{10}$ and caring for patients' psychosocial and spiritual needs is not the usual goal in the ED. However, studies have shown that implementation of EOL pathways increases the knowledge and confidence of nursing personnel in managing dying patients and leads to consistent and improved patient care. ${ }^{11-13}$ Moreover, palliative care in terminally ill patients has been shown to reduce hospitalisation costs. ${ }^{14}$ Novel initiatives for EOL care education for ED healthcare staff and clinical guidelines for delivery of EOL care have been implemented in several Singapore public hospitals. These initiatives serve to enhance the quality of EOL care provision for imminently dying patients and their grieving family members. Effective EOL programmes improve patient-provider communication, provide comfort and relief of burdensome symptoms in terminally ill patients, pay attention to spiritual needs, maintain patients' dignity and respect family members' social concerns during the last hours or days of life. ${ }^{15}$

Since the progressive initiation of the ED EOL programme in Singapore since 2014, its impact on quality improvement has yet to be determined.

We propose this multicentre interrupted time series study to improve the quality of EOL care for actively dying patients in the ED. The specific objectives in this study are to:

- Systematically measure the current quality of EOL care in three Singapore EDs to identify gaps in management.
- Formulate improvements needed to address these gaps and implement the improved EOL care protocol.

- Measure (a) the quality of care postimplementation of the improved EOL care and (b) the impact on health services utilisation and associated costs.

\section{METHODS/DESIGN}

\section{Study design}

This is a quasiexperimental interrupted time series study conducted in the EDs of three public hospitals (National University Hospital (NUH), Changi General Hospital $(\mathrm{CGH})$ and Khoo Teck Puat Hospital (KTPH)) in Singapore to evaluate the quality of EOL care rendered with measures conducted postimplementation of interventions to assess improvement in EOL care. The project is overseen by biostatisticians and epidemiologists from the Singapore Clinical Research Institute and site investigators from participating hospitals. The study comprises five phases: prephase 1, pilot phase and phases 1-3. Apart from prephase 1 which is retrospective, all other phases are conducted prospectively. Details of each phases with variables and data to be collected are detailed below.

\section{Study setting and sites}

The public hospitals included in this study, namely NUH, CGH and KTPH, belong to the three main healthcare clusters in Singapore - the National University Health System, Singapore Health Services and National Healthcare Group, which serve the country's western, eastern and northern populations, respectively. ${ }^{16}$ Each of the three hospitals are tertiary centres with annual ED census of more than 100000 attendances.

Currently in our local healthcare setting, patients who are at their EOL phase may be managed at the following locations: in their own homes with a dedicated caregiver and home hospice services, in inpatient hospice facilities or in acute care hospitals. The healthcare cost and resources incurred will thus depend on the setting where EOL care is provided.

\section{Prephase 1 (retrospective)}

In prephase 1, we aim to determine the baseline data prior to any implementation of EOL protocols in the respective hospitals to facilitate the assessment of the impact on health services utilisation. Using the hospital database, patients who died within 5 days of ED attendance will be identified. This timeframe is chosen based on unpublished data from a previous study done in NUH, which showed that EOL patients who were admitted have a median length of inpatient stay of 1.4 days with an IQR of 0.4-5.2 days. ${ }^{17}$ Chart reviews will be performed to determine if the patient fulfilled the criteria for EOL management. Information on premorbid conditions, any prior referral to palliative services, clinical management, health services utilisation and associated costs will be collected. The period of the chart reviews are as follows:

- NUH: 1 January 2013 to 31 December 2013.

- CGH: 1 July 2016 to 30 June 2017. 
- KTPH: 1 July 2016 to 30 June 2017.

These periods of chart reviews correspond to the 12 months that preceded initiation of any EOL initiative in the EDs of the respective hospitals. Being in the forefront of the public healthcare sector, any patient can present to the EDs in Singapore, either by self-referral/ walk-in, ambulance or referral by another healthcare facility. We often encounter patients who are actively dying from their underlying comorbidities or from a sudden catastrophic event. Before the initiation of any EOL care pathway in each of the EDs, management of symptoms in any ED patients who are actively dying were all based on attending physicians' discretion. For instance, an EOL patient with increased secretions may not be routinely given hyoscine butylbromide for relief. Recognising this gap, each of the three EDs have independently established their own workflows for a protocolised care bundle to manage such patients, so that EOL symptoms can be more effectively managed based on an established pathway. Despite this, an observational study in NUH showed that there is room for EOL care to be improved and optimised. ${ }^{17}$

\section{Pilot phase (January-April 2019)}

The pilot phase tests the face and construct validity and reliability of a newly developed questionnaire for measuring the quality of EOL care in EDs in the Asian context. The questionnaire development takes reference from the Care of the Dying Evaluation (CODE).$^{18}$ Participants, who are family members of EOL patients, will be requested to complete the newly developed questionnaire (renamed Care of the Dying Evaluation - Emergency Medicine (CODE-EM)) and then interviewed about their experience of completing the questionnaire and to participate in a retest of the questionnaire 1 month later either by phone or mail. The postquestionnaire interview aims to examine the validity of the questionnaire in terms of language, length, timing and relevance.

\section{Phase 1 (April 2019-April 2020 projected)}

At the start of this study, all three hospitals have EOL workflows in place that was developed within each department. A brief summary and comparison of the three workflows are illustrated in figure 1 . In phase 1 of the study, quality indicators will be measured prospectively to evaluate the aspects of EOL care rendered to patients under existing workflows. Data of recruited patients from the start of study enrolment until demise or terminal discharge from the hospital will be collected. Their electronic medical records and ED consult notes will be reviewed from time of ED attendance until terminal discharge or death to assess their inpatient progress and discharge outcomes. Apart from chart reviews of the EOL patients, the validated CODE-EM questionnaire will also be conducted with the nominated caregivers of these patients. The quality indicators will be divided into patient-centred, family-centred and staff-centred outcomes.

The patient-centred outcomes to be evaluated include proportion of patients who fits EOL criteria and died within 5 days, proportion of patients on EOL pathway who have documentation that patient and/or family
NUH ED

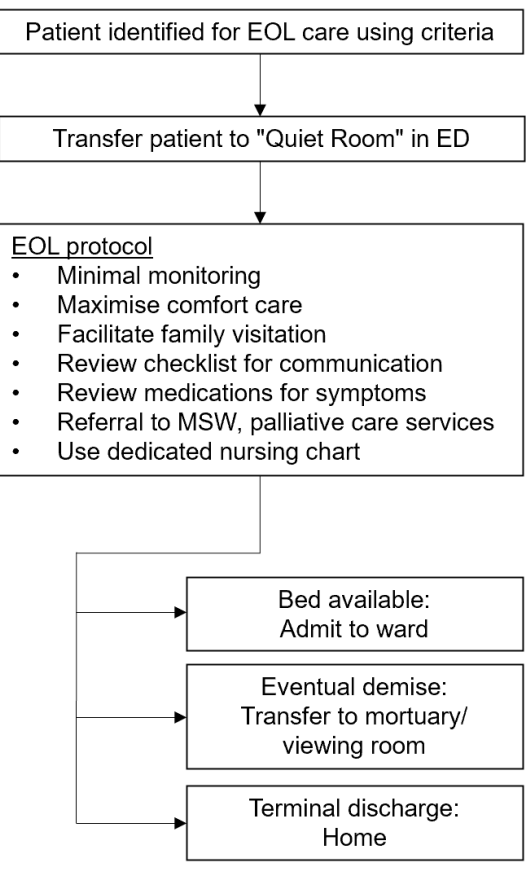

CGH ED

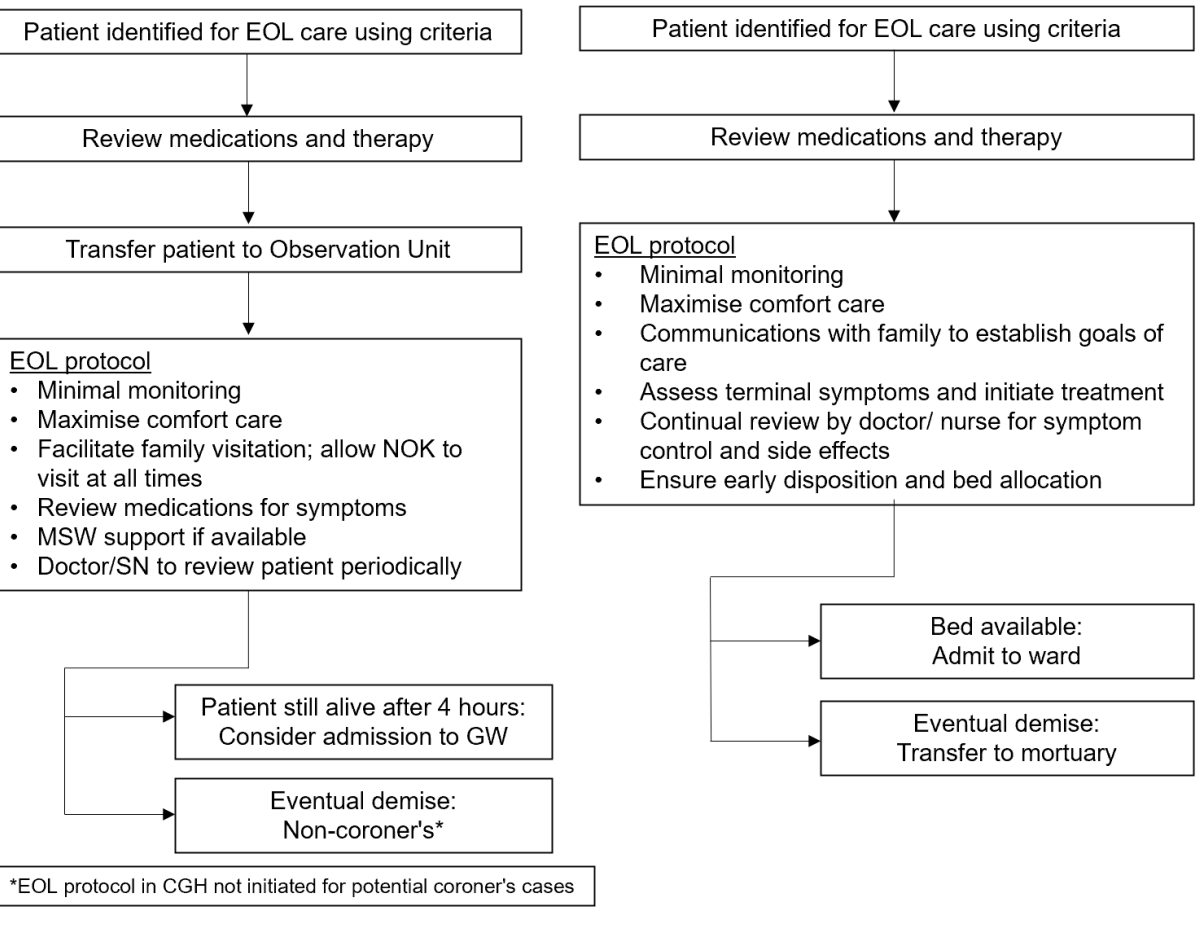

Legend: ED - emergency department; EOL - end-of-life; GW - general ward; MSW - medical social worker; NOK - next-of-kin; SN - staff nurse

Figure 1 EOL care workflow in the emergency departments of National University Hospital (NUH), Changi General Hospital (CGH) and Khoo Teck Puat Hospital (KTPH). 
are given opportunities to discuss an individualised care plan and proportion of patients with symptoms who are prescribed with medicines with individualised indications for use, dosage and route of administration.

In addition, the following data will be collected for all recruited EOL patients: demographic information, clinical presentation, comorbidities, healthcare utilisation, interventions post-EOL, length of hospitalisation, communications with the family, comfort care measures, cost of hospitalisation, prior do-not-resuscitate decisions, disposition (death, terminal discharge home or hospice and admission to inpatient unit) and cause of death.

Family-centred indicators are evaluated using the validated CODE-EM questionnaire (see online supplementary material 1) to assess the quality of care and the level of support provided to patients and their families in the ED. The key indicators include proportion of participants who perceived that their family member was treated with dignity and respect by doctors and nurses and the proportion of participants who perceived that they were adequately supported.

For staff-centred indicators, we aim to determine the level of healthcare providers' knowledge and perception of the EOL care using a locally designed anonymous survey (see online supplementary material 2) conducted monthly in the first 3 months, followed by 6 and 12 months later. This survey will be self-administered. Information regarding the work experience, existing level of palliative care training and designation will also be collected. The main components to be evaluated are level of confidence in communications and providing emotional support, knowledge, clarity of nurses' and doctors' roles, conduciveness of physical environment and satisfaction of overall care quality rendered in managing EOL patients in the ED.

\section{Phase 2 (March-April 2020 projected)}

Phase 2 will commence at the end of phase 1 based on the results obtained and will encompass focus group discussions (FGDs), revision of EOL care components and implementation of improved EOL care in the three institutions. There will be separate FGDs for healthcare professionals, family members and primary care providers (such as general practitioners and home hospice healthcare workers) conducted to identify the gaps for improvement and areas that were done well in the existing EOL care paths. Recommendations to improve the quality of the existing EOL care will also be sought.

By combining the qualitative and quantitative results of phases 1 and 2, the study investigators from the three hospitals will assimilate all data and revise the current protocol accordingly. Combined and in-house staff training on palliative care and improved EOL management workflow will ensue thereafter.

\section{Phase 3 (May 2020 to -May 2021 projected)}

In phase 3, new and improved EOL workflows will be rolled out in all three hospitals. The quality indicators and outcomes to be evaluated will be the same as in phase 1 (as detailed above). The results obtained in phase 3 will then be compared with that in phase 1 . Staff-centred indicators will be mapped against the combined and in-house training sessions to evaluate for any changes.

\section{Project materials}

Permission was obtained from the original developers of the CODE-EM questionnaire to adapt it for local and ED contexts (see online supplementary material 1 ), which was validated during the pilot phase. To evaluate staffcentred indicators, a locally designed staff survey was used (see online supplementary material 2). The staff survey was developed by ED attendings and nurses trained in palliative care for the purpose of evaluating quality of care rendered during the initiation of the EOL workflow in its early stages in NUH in 2013. The staff survey was piloted and improved on during those initial stages, not within the study period.

\section{Patient selection}

All the following inclusion criteria must be fulfilled:

- Actively dying patient or high likelihood of mortality within this admission (based on attending physician's judgement using available clinical data such as vital signs, mental status and biochemical or radiological investigations, if available).

- Family accepts that the goals of care are provision of comfort, symptom relief and respect of dignity.

- Patient is not a candidate for cardiopulmonary resuscitation, endotracheal intubation or transfer to the intensive care unit.

- Any of the life-limiting conditions: chronic frailty with poor functional state and limited reversibility (Karnofsky Performance Scale $<40 \%)^{19}$; chronic severe illness with poor prognosis (terminal cancer, end-stage renal failure (refusal or withdrawal of dialysis), end-stage respiratory, heart or liver disease, advanced neurological disease including dementia, Parkinson's disease and severely disabling stroke); or acute severe catastrophic conditions and at risk of dying with complications that are not reversible, as subject to the treating clinician's judgement.

We excluded the following subjects: vulnerable population (eg, prisoners and pregnant women); refusal to participate; patients who have been recruited, or had declined participation, during the previous ED attendance(s); patients in periarrest state; and/or family members who are not available or present at the patient's bedside.

\section{Patient and public involvement}

There was no patient or public involvement in the design and conduct of this study.

\section{Sample size calculation}

Preliminary data from 2014 to 2017 in NUH for calculation indicated an average of seven patients who fulfilled the EOL criteria in NUH every month. We expect to receive 
approximately 15 eligible EOL patients per month from the three centres and about 195 eligible patients in phase 1 (13 months) and phase 3 (13 months), respectively.

Based on NUH's existing data, the current correct identification rate is $24.4 \%$. We performed a power analysis to estimate the expected power with various combinations of recruited subjects and observed improvement. We could achieve an $80 \%$ power with an alpha of 0.05 for a two-sided test, if we were to:

1. Recruit 200 subjects for each phase and expected to observe an absolute difference $\geq 13 \%$.

2. Recruit 180 subjects for each phase and expected to observe an absolute difference $\geq 14 \%$.

3. Recruit 160 subjects for each phase and expected to observe an absolute difference $\geq 15 \%$.

4. Recruit 140 subjects for each phase and expected to observe an absolute difference $\geq 16 \%$.

\section{Outcomes of interest and statistical analyses}

Pilot phase analysis planning

The postquestionnaire interviews about the experience of completing the questionnaire will be transcribed verbatim and analysed using a content analysis framework. Data will be refined into specific categories, with words and phrases of shared meaning. A randomised selection of interview transcripts will be independently reviewed by a second investigator, not directly involved in the data collection, to check for coding, and any discrepancies will be discussed with a third investigator. The stability of the developed questionnaire over time will be assessed using the following measures: percentage agreement, $\kappa$ statistic (Cohen's for nominal response options and weighted for ordinal response options) and Spearman's correlation coefficient (for ordinal data). The criteria for good stability over time is defined as percentage agreement $>70 \%, \kappa>0.6$ and $r>0.7$ and moderate stability over time as percentage agreement $>30 \%, \mathrm{k}>0.40$ and $\mathrm{r}>0.3$. Confirmatory factor analysis will be used to assess construct validity. The suitability of questions will be examined by inspection of the correlation matrix and the Goodness of Fit Index.

\section{Statistical analysis for other phases}

Descriptive statistics will be obtained about the baseline characteristics of participants. Continuous variables will be presented as mean $\pm \mathrm{SD}$, while categorical variables will be reported as absolute numbers and percentages. Differences regarding outcomes will be analysed using independent samples t-test or Mann-Whitney U test, or paired t-test for pre-EOL and post-EOL measurements. Symptom burden (eg, differences in frequency of symptoms) and CODE-EM questionnaire will be calculated.

Time series regression will be used to analyse the results from staff survey, using seven data points (ie, collected at every 2 months) from each phase. The best fit preprogramme and postprogramme revision lines will be estimated by using linear regression, and autocorrection will be adjusted for by using maximum likelihood methods where appropriate. The change in the slopes of the regression lines will be estimated. Data will be analysed using Stata V.15.

\section{Health economic impact analysis}

This study will also evaluate the financial viability of EOL care by conducting a health economic evaluation from a hospital system perspective. The costing for our health economic evaluation has two components. First, for costs directly related to services to patients, itemised data and information on resource utilisation associated with patient management will be recorded for each participant, including initial management in ED, services associated with protocol implementation and follow-up. Second, costs related to protocol implementation including extra administration and medical staff will be evaluated as well, according to the required staff levels and time needed. The potential impact of protocol implementation on medical and administrative management resources will be studied by comparing with historical data from chart reviews. After identifying the extra care components associated with study protocol implementation (eg, accessing the on-call palliative care specialist), for base case analysis, private rates of each service will be applied to calculate the average cost and savings. In Singapore, the billing in our public hospitals can be broadly categorised into 'private rates' and 'subsidised rates' (after receiving government subsidies). We will use 'private rate' of each service item to best estimate the costs.

The health economic analysis will consist of two components: (1) a cost-benefit analysis to assess the cost-benefit ratio, which will be presented as 'for every 1 extra dollar spent on the protocol implementation, how much savings can be realised by the potential reduction in hospital admissions and length of hospital stay'; and (2) a costeffectiveness analysis to estimate the incremental costeffectiveness ratio. To address possible variations in patient medical conditions and the outcomes, such as variations in compliance, and length of hospital stay, a series of oneway sensitivity analyses will be conducted to address the impact of each parameter's uncertainty and assess the robustness of study estimations for generalisability.

\section{Ethics and dissemination}

Ethics approval for this study was obtained from the National Healthcare Group Domain Specific Review Board (DSRB reference no: 2018/00838) for waiver of informed consent for the retrospective cohort in prephase 1. Written informed consent will be obtained from all study participants in all other phases. For EOL patients who have the mental capacity for informed consent, their consent for participation will be sought and will not be overridden by their family members. For patients who have impaired cognition, informed consent will be sought from their legally acceptable representative in accordance to DSRB's guidelines. The findings from this study will be disseminated locally and internationally through manuscript publications in peer-reviewed 
journals and conference presentations at national and international platforms.

\section{DISCUSSION}

To the best of our knowledge, this is the first multicentre study using both qualitative and quantitative methods and focusing on evaluation of EOL care provided in the ED. Additionally, apart from focusing on patients with chronic irreversible illnesses such as advanced cancer, our study cohort includes other death trajectories such as sudden death due to an acute catastrophic event. This is an area where information on palliative care is lacking.

Our study design has distinct advantages over the traditional epidemiological cohort (before and after) and case-control designs that are limited by confounding and lack of usefulness in intervention studies. The interrupted time series study design would be able to detect changes that are delayed or intermittent and can assess if the change is permanent or temporary. The design is simpler without the need for randomisation, which is not possible in EOL research. There is also the ability to control for confounding variables and regression to the mean.

Furthermore, our study design allows us to obtain both qualitative and quantitative knowledge with regard to current quality of care and identify areas for improvement. The information obtained from intimate FGDs with the family members and our community healthcare partners would be consequential in providing in-depth information on the current quality of care and difficulties faced in order to guide changes in protocols and policies. The unique setting of a multicultural Singapore society may also provide some insights into previously underinvestigated cultural beliefs and values that can be a focus of future qualitative studies in the region.

Despite the strengths of our study, the design presents some inherent limitations. First, the chart reviews of the retrospective cohort in prephase 1 may have information bias. We aim to mitigate this by looking through all available paper and electronic medical records to reduce missing information. Second, the response rates from questionnaires and staff surveys may also pose a challenge. In order to encourage participation, small tokens of appreciation and incentives will be given out to study participants. Third, the use of non-probability sampling and enrolment when research assistants are around during office hours may also introduce a degree of selection bias. This is however necessary due to economic constraints that preclude round-the-clock staffing of research personnel. However, we have mitigated this by screening through ED attendances beyond office hours and over the weekend and contacting eligible family members in the wards whenever appropriate for invitation to participate.

In conclusion, the results of our study would have the potential to identify current barriers and inform emergency physicians globally on the best practices in providing EOL care in the ED for patients who are at the last stages of their lives from a myriad of illnesses.

Author affiliations

${ }^{1}$ Emergency Medicine Department, National University Hospital, National University Health System, Singapore

${ }^{2}$ Department of Surgery, Yong Loo Lin School of Medicine, National University of Singapore, Singapore

${ }^{3}$ Accident \& Emergency Medicine, Changi General Hospital, Singapore

${ }^{4}$ Acute and Emergency Care Centre, Khoo Teck Puat Hospital, Singapore

${ }^{5}$ Singapore Clinical Research Institute, Singapore

${ }^{6}$ Department of Medicine, Yong Loo Lin School of Medicine, National University of Singapore, Singapore

${ }^{7}$ Division of Palliative Care, National University Cancer Institute, Singapore

Contributors RYP conceived the study, designed the final study protocol, provided the domain knowledge expertise and is the principal investigator of the grant; WSK helped in the design of the final study protocol, contributed to the technical design and revised the initial manuscript draft; LT and RK helped in the design of the final study protocol and are coordinating site investigators; YKYW, LS and CQZ contributed to the technical design and provided biostatistical and epidemiological support; JL, SL, UCS, WCY and NGCC provided clinical input and perspectives to the qualitative aspects of the study; MTC helped in the design of the final study protocol, drafted the initial manuscript and coordinated ethics approval; II helped in the design of the final study protocol, had a part in the technical design and coordinated collaboration among investigators from all institutions. All authors read and approved the final manuscript.

Funding This work is supported by a grant from the National Medical Research Council Health Services Research Grant (grant number: MOH-HSRGEoL17Ju-0001). The funding body provides funds for employment of research assistants, engagement of statistical support from Singapore Clinical Research Institute and pays for all study-related expenses such as reimbursements of study participants and manuscripts processing fees. It is not involved in the design of the study, and collection, analysis and interpretation of data, and in writing of the manuscript.

Competing interests The authors declare that they have no competing interests. This study is funded by a major government funding body (National Medical Research Council, Ministry of Health, Singapore), and there is no funding or assistance from any commercial organisation.

Patient and public involvement Patients and/or the public were not involved in the design, or conduct, or reporting, or dissemination plans of this research.

Patient consent for publication Not required.

Ethics approval Ethics approval for this study was obtained from the National Healthcare Group Domain Specific Review Board (DSRB reference no: 2018/00838) for waiver of informed consent for the retrospective cohort in pre-Phase 1. Written informed consent will be obtained from all study participants in all other phases.

Provenance and peer review Not commissioned; externally peer reviewed.

Open access This is an open access article distributed in accordance with the Creative Commons Attribution Non Commercial (CC BY-NC 4.0) license, which permits others to distribute, remix, adapt, build upon this work non-commercially, and license their derivative works on different terms, provided the original work is properly cited, appropriate credit is given, any changes made indicated, and the use is non-commercial. See: http://creativecommons.org/licenses/by-nc/4.0/.

\section{ORCID iDs}

Win Sen Kuan http://orcid.org/0000-0002-2134-7842

Mui Teng Chua http://orcid.org/0000-0002-6326-4914

\section{REFERENCES}

1 Field MJ, Cassel CK. Approaching death: improving care at the end of life. N Engl J Med 1998;339:274-5.

2 Stjernswärd J, Foley KM, Ferris FD. The public health strategy for palliative care. J Pain Symptom Manage 2007;33:486-93.

3 Tang ST. When death is imminent: where terminally ill patients with cancer prefer to die and why. Cancer Nurs 2003;26:245-51.

4 Gloss K. End of life care in emergency departments: a review of the literature. Emerg Nurse 2017;25:29-38. 
5 Smith AK, McCarthy E, Weber E, et al. Half of older Americans seen in emergency department in last month of life; most admitted to Hospital, and many die there. Health Aff 2012;31:1277-85.

6 Krikorian A, Limonero JT, Maté J. Suffering and distress at the endof-life. Psychooncology 2012;21:799-808.

7 Beckstrand RL, Wood RD, Callister LC, et al. Emergency nurses' suggestions for improving end-of-life care obstacles. J Emerg Nurs 2012;38:e7-14.

8 Kistler EA, Sean Morrison R, Richardson LD, et al. Emergency department-triggered palliative care in advanced cancer: proof of concept. Acad Emerg Med 2015;22:237-9.

9 Lien centre for palliative care, Duke-NUS graduate medical school. Report on the National strategy for palliative care, 2011. Available: https://www.singaporehospice.org.sg/en/wp-content/uploads/2018/ 08/Report_on_National_Strategy_for_Palliative_Care-5Jan2012.pdf [Accessed 13 June 2017].

10 Chan GK. End-Of-Life models and emergency department care. Acad Emerg Med 2004;11:79-86.

11 Selman L, Robinson V, Klass L, et al. Improving confidence and competence of healthcare professionals in end-of-life care: an evaluation of the 'Transforming End of Life Care' course at an acute hospital trust. BMJ Support Palliat Care 2016;6:231-6.

12 Luhrs CA, Penrod JD. End-Of-Life care pathways. Curr Opin Support Palliat Care 2007;1:198-201.
13 Weng T-C, Yang Y-C, Chen P-J, et al. Implementing a novel model for hospice and palliative care in the emergency department. Medicine 2017;96:e6943.

14 Aldridge MD, Kelley AS. The myth regarding the high cost of end-oflife care. Am J Public Health 2015;105:2411-5.

15 Threapleton DE, Chung RY, Wong SYS, et al. Care toward the end of life in older populations and its implementation facilitators and barriers: a scoping review. J Am Med Dir Assoc 2017;18:1000-9.

16 Poon $\mathrm{CH}$. Public healthcare sector to be reorganised into 3 integrated clusters, new polyclinic group to be formed. The Straits Times, 2017. Available: https://www.straitstimes.com/singapore/ health/public-healthcare-sector-to-be-reorganised-into-3-integratedclusters-new [Accessed 16 October 2019].

17 Chor WPD, Wong SYP, Ikbal MFBM, et al. Initiating end-of-life care at the emergency department: an observational study. Am J Hosp Palliat Care 2019;36:941-6.

18 Mayland CR, Lees C, Germain A, et al. Caring for those who die at home: the use and validation of 'Care Of the Dying Evaluation' (CODE) with bereaved relatives. BMJ Support Palliat Care 2014;4:167-74.

19 Karnofsky D, Burchenal J. The clinical evaluation of chemotherapeutic agents in cancer.. Eval Chemother agen ts 1949:191-205. 